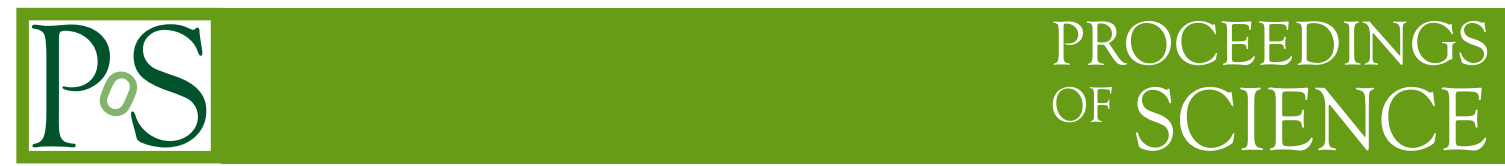

\title{
QCD tests at NA48/2 experiment
}

\author{
Gianluca Lamanna*t \\ Scuola Normale Superiore \& INFN Pisa - Italy \\ E-mail: gianluca.lamanna@cern.ch
}

The main goal of the NA48/2 experiment was to measure the CP violating asymmetry in the charged kaon decay in three pions. Thanks to the very high statistics achieved both in $\mathrm{K}^{+}$and $K^{-}$decays, many other interesting measurements has been performed with unprecedented precision. The analysis of the strong interaction dynamics at low energy is one of the main issues studied using kaon decays. In particular, in this paper, the results obtained in $\pi-\pi$ scattering lengths measurement will be presented using two different approaches, and the Branching Ratio measurements of $K^{+} \rightarrow \pi^{+} \gamma \gamma$ and $K^{+} \rightarrow \pi^{+} e^{+} e^{-} \gamma$ that are sensitive tests of Chiral Perturbation Theory (ChPT).

European Physical Society Europhysics Conference on High Energy Physics, EPS-HEP 2009, July 16 - 222009

Krakow, Poland

\footnotetext{
*Speaker.

$\dagger$ On behalf of the NA48/2 collaboration.
} 


\section{Experimental setup}

The NA48/2 experiment beam line has been designed to measure the CP violating charged asymmetry in the $K \rightarrow 3 \pi$ decay [1]. Simultaneous positive and negative kaons beams are produced in the same beryllium target by impinging $400 \mathrm{GeV}$ protons from the CERN/SPS accelerator. The momentum range of $(60 \pm 3) \mathrm{GeV} / \mathrm{c}$ is selected, for both beams, in the first "achromat" and a complex system of magnets allows to have superimposed and focused beams $\sim 200 \mathrm{~m}$ downstream, at the end of the $\sim 100 \mathrm{~m}$ long decay region. A schematic view of the beam line can be found in [1]. Both $K^{+}$and $K^{-}$decays are collected in the same NA48 detectors, described elsewhere [2]. The $K_{e 4}$ analysis is essentially based on the magnetic spectrometer, consisting of a magnet dipole and two sets of two drift chambers with a momentum resolution of $\sigma(p) / p=(1.0+0.044 \cdot p) \%$ (with $\mathrm{p}$ in $\mathrm{GeV} / \mathrm{c}$ ). The $K^{ \pm} \rightarrow \pi^{ \pm} \pi^{0} \pi^{0}$ analysis uses the electromagnetic calorimeter to identify the gammas produced by the $\pi^{0}$ decay. The E.M. calorimeter has $\sim 27$ radiation lengths of liquid krypton (LKr) with a resolution of $\sigma(E) / E=(3.2 / \sqrt{E} \oplus 9.0 / E \oplus 0.42) \%$ (with $\mathrm{E}$ in $\mathrm{GeV}$ ). The BR measurements, presented in this paper, use both $\mathrm{LKr}$ and spectrometer.

\section{2. $K_{e 4}$ decay analysis}

The $K^{ \pm} \rightarrow \pi^{+} \pi^{-} e^{ \pm} v\left(K_{e} 4\right)$ kinematics is fully described using the 5 Cabibbo-Maksymowicz variables [3] : the dilepton and dipion invariant mass and the three angles $\Theta_{\pi}, \Theta_{e}$ and $\Phi$, as defined in fig.1. Thanks to these variables the two axials $(F, G)$ and one vectorial $(H)$ form factors contributing to the transition amplitude, are written in terms of a partial wave expansion [丹]:

$$
F=F_{s} e^{i \delta_{s}}+F_{p} e^{i \delta_{p}}+\ldots, \quad G=G_{p} e^{i \delta_{p}}+\ldots, \quad H=H_{p} e^{i \delta_{p}}+\ldots
$$

the four form factors can be further expanded in powers of $q^{2}=\left(M_{\pi \pi}^{2} / 4 m_{\pi}^{2}\right)-1$ :

$$
F_{s}=\left(f_{s}+f_{s}^{\prime} q^{2}+f_{s}^{\prime \prime} q^{4}\right), \quad F_{p}=f_{p}, \quad G_{p}=\left(g_{p}+g_{p}^{\prime} q^{2}\right), \quad H_{p}=h_{p}
$$

and only the phase shift $\delta\left(q^{2}\right)=\delta_{s}-\delta_{p}$ is taken into account. About 677500 decays were selected looking for events in which three good reconstructed tracks are identified in the magnetic spectrometer. The particle identification, to distinguish between electron and pions, exploits the ratio between the energy measured in the calorimeter and the momentum in spectrometer (that is 1 for electrons). The background, coming mainly from $K^{ \pm} \rightarrow \pi^{ \pm} \pi^{+} \pi^{-}$decay with a pion misidentified as electron or with a pion decay $\pi \rightarrow e v$, is evaluated by studying the "wrong" sign events, i.e. the events for which the $\Delta S=\Delta Q$ rule is violated (we are not sensitive to measure this violation). The total background is at level of $0.5 \%$. In order to fit the form factors and the phase shift, the whole data sample has been subdivided in 15000 bins defined in the Cabibbo-Maksymowicz variables 5 dimensional space, and a GEANT3 [5] based montecarlo has been employed. In particular in each of the 10 bins along $M_{\pi \pi}$ a 4 parameters fit was performed in order to extract the form factors. In tab. 11 the results are summarized. The agreement between data and Montecarlo distributions is very good for each of the variables used to define the form factors.

With respect to the results already presented in [6] on a partial data set, in this analysis a restricted kaon momentum range $[54,66] \mathrm{GeV} / \mathrm{c}$ in the selection has been applied in order to minimize 


$$
\mid \begin{array}{lc|lc}
f_{s}^{\prime} / f_{s}= & 0.0152 \pm 0.007 \pm 0.005 & g_{p} / f_{s}= & 0.0868 \pm 0.010 \pm 0.010 \\
f_{s}^{\prime \prime} / f_{s}= & -0.073 \pm 0.007 \pm 0.006 & g_{p}^{\prime} / f_{s}= & 0.089 \pm 0.017 \pm 0.013 \\
f_{e}^{\prime} / f_{s}= & 0.068 \pm 0.006 \pm 0.007 & h_{p} / f_{s}= & -0.398 \pm 0.015 \pm 0.008 \\
f_{p} / f_{s}= & -0.048 \pm 0.003 \pm 0.004 & &
\end{array}
$$

Table 1: Preliminary results with statistical and systematics error

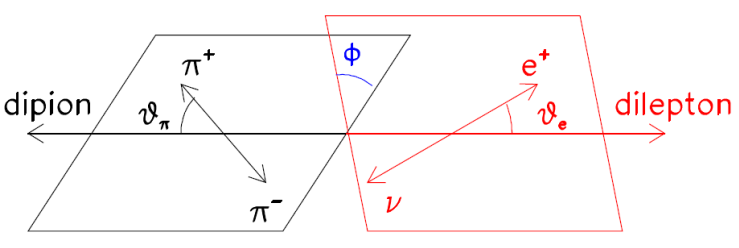

Figure 1: Topology of the $K_{e 4}$ decay and defininition of the Cabibbo-Maksymowicz variables.

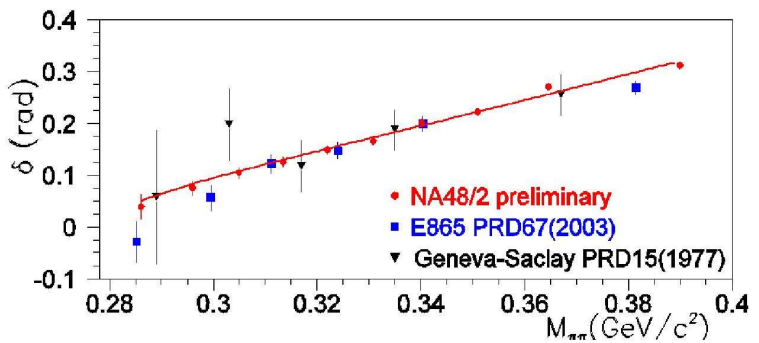

Figure 2: Phase shift as a function of $M_{\pi \pi}$. The precision in the 10 points measured by NA48/2 exceeds the previous measurements.

the background contamination. A second fit is made to determine the form factors $q^{2}$ dependence. In the case of the phase shift $\delta$, the Roy equations [7], simply based on analicity and unitarity, are used to fit the $q^{2}$ dependence. Through the Roy equations, after an extrapolation from $\pi \pi$ scattering data at higher energy, it is possible to correlate the $\delta$ phase shift to the scattering lengths $a_{0}$ and $a_{2}$. Using the numerical solutions of the Roy equations (as calculated by several groups [8] [9]), the isospin breaking corrections have to be included in order to extract the corrected value for the $\pi-\pi$ scattering lengths [10]. In fig.2] the phase shift dependence on $M_{\pi \pi}$ is shown as fitted with the Roy equations. The extracted values for the scattering lengths are:

$$
\begin{gathered}
a_{0} m_{\pi}=0.2220 \pm 0.0128_{\text {stat }} \pm 0.0050_{\text {syst }} \pm 0.0037_{\text {th }} \\
a_{2} m_{\pi}=-0.0432 \pm 0.0086_{\text {stat }} \pm 0.0034_{\text {syst }} \pm 0.0028_{\text {th }}
\end{gathered}
$$

with a 97\% correlation coefficient. Using the relation among $a_{0}$ and $a_{2}$ predicted by ChPT [12] the value from the 1-parameter fit is:

$$
a_{0} m_{\pi}=0.2206 \pm 0.0049_{\text {stat }} \pm 0.0018_{\text {syst }} \pm 0.0064_{\text {th }}
$$

The present world average on the pionic scattering lengths from $K_{e 4}$, dominated by the results shown here, is:

$$
\begin{gathered}
a_{0} m_{\pi}=0.2199 \pm 0.0125_{\exp } \pm 0.0037_{t h} \\
a_{2} m_{\pi}=-0.0430 \pm 0.0083_{\exp } \pm 0.0028_{t h}
\end{gathered}
$$




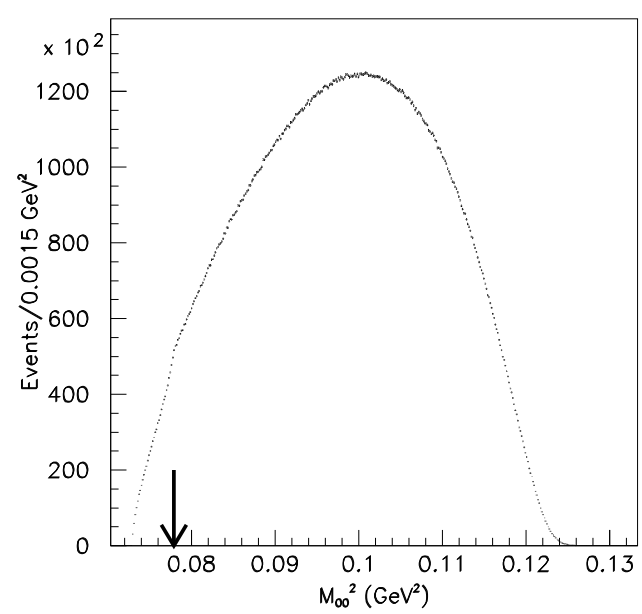

Figure 3: $M_{\pi^{0} \pi^{0}}^{2}$ mass distribution in $K^{ \pm} \rightarrow \pi^{ \pm} \pi^{0} \pi^{0}$. The arrow indicated the cusp position at $2 m_{\pi \pi}$.

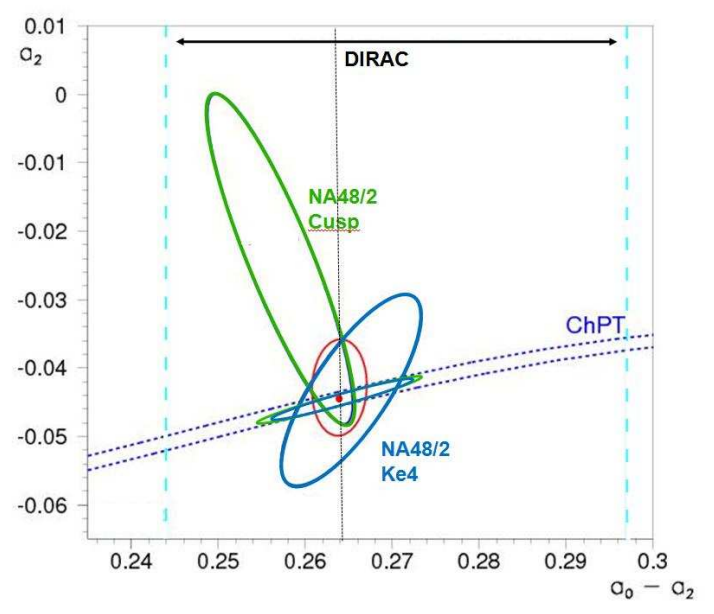

Figure 4: Cusp and Ke4 NA48/2 results. Comparison with theoretical predictions given by ChPT and experimental result given by DIRAC experiment [11] (based on pionium lifetime analysis).

\section{Cusp analysis}

The pion scattering lengths can also be measured through the study of the "cusp" in the $\pi^{0} \pi^{0}$ invariant mass distribution $\left(M_{00}^{2}\right)$ in $K^{ \pm} \rightarrow \pi^{ \pm} \pi^{0} \pi^{0}$ decay. As shown in fig. 3, at $2 m_{\pi}$ a singularity (cusp) appears; this cannot be explained by a simple polynomial expansion of the matrix element (as used in [13]). The interpretation of this structure was given by [14] as due to the $\pi^{+} \pi^{-} \rightarrow$ $\pi^{0} \pi^{0}$ strong rescattering, having different real and imaginary behaviour below and above the $2 \pi^{+}$ production threshold. Several theoretical approachs (for instance [15] [16]) has been implemented in order to exploit the experimental precision obtained thanks to the sizeable statistics collected (more than $\sim 60 \cdot 10^{6} K^{ \pm} \rightarrow \pi^{ \pm} \pi^{0} \pi^{0}$ ) and the excellent $m_{\pi^{0} \pi^{0}}$ mass resolution. In particular we use two different models to fit our data: the Cabibbo-Isidori (CI) [17] and the Bern-Bonn (BB) [18] . The results are in good agreement in spite of the fact that they are based on different hypothesis (the BB results are shown here, due to the better $\chi^{2}$ and the most complete theory [19]):

$$
\begin{gathered}
\left(a_{0}-a_{2}\right) m_{\pi}=0.2571 \pm 0.0048_{\text {stat }} \pm 0.0025_{\text {syst }} \pm 0.0014_{\text {ext }} \\
a_{2} m_{\pi}=-0.024 \pm 0.013_{\text {stat }} \pm 0.009_{\text {syst }} \pm 0.002_{\text {ext }}
\end{gathered}
$$

Using the same ChPT constraint given by [12] mentioned above, the 1 parameter fit gives:

$$
\left(a_{0}-a_{2}\right) m_{\pi}=0.2633 \pm 0.0024_{\text {stat }} \pm 0.0014_{\text {syst }} \pm 0.0019_{\text {ext }}
$$

These results are in agreement with our previous results based on a partial set of data [20].

\section{Comparison between Ke4 and Cusp analysis}

Two different approaches to measure the pion scattering lengths has been presented. From an experimental point of view the two processes, the $K^{ \pm} \rightarrow \pi^{+} \pi^{-} e^{ \pm} v$ and the $K^{ \pm} \rightarrow \pi^{ \pm} \pi^{0} \pi^{0}$, are 


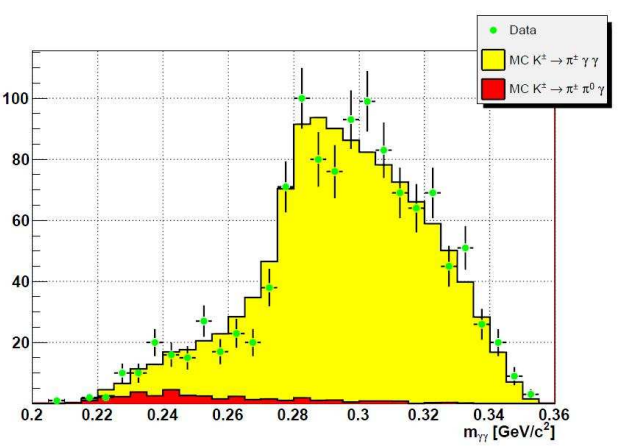

Figure 5: The $m_{\gamma \gamma}$ data distribution is well described by ChPT for defined values of the c parameter at $O\left(p^{6}\right)$

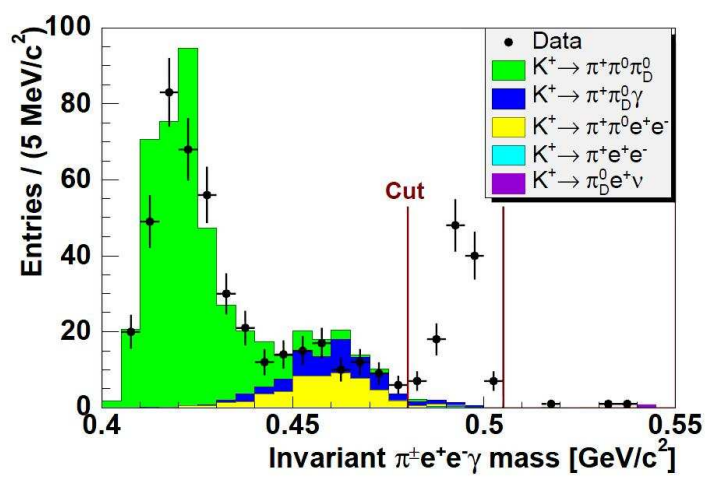

Figure 6: The background contribution below the signal peak is $6.1 \%$ (mainly due to $K^{+} \rightarrow \pi^{+} \pi^{0} D \gamma$ decay

collected by different sub-detectors with different contribution to the systematics uncertainty. On the other hands the theoretical inputs used to extract informations from the decays dynamics are totally different: based on Roy equations and isospin correction in the Ke4 case and on final state strong rescattering or on ChPT theory in the Cusp case. In spite of these differences the results obtained are in good agreement, as shown in fig. 4 . The combined results:

$$
\begin{gathered}
\left(a_{0}-a_{2}\right) m_{\pi}=0.2639 \pm 0.0020_{\text {stat }} \pm 0.0004_{\text {syst }} \\
a_{2} m_{\pi}=-0.0429 \pm 0.0044_{\text {stat }} \pm 0.0016_{\text {syst }}
\end{gathered}
$$

Assuming the ChPT constraint:

$$
\left(a_{0}-a_{2}\right) m_{\pi}=0.264 \pm 0.0020_{\text {stat }} \pm 0.0017_{\text {syst }}
$$

These results are in perfect agreement with the ChPT prediction [12] :

$$
\left(a_{0}-a_{2}\right) m_{\pi}=0.265 \pm 0.004
$$

\section{Other QCD tests}

The kaon decays are an excellent laboratory to test the low energy strong interaction perturbative theories, as the ChPT. For example, the NA48/2 measurement of the $K^{+} \rightarrow \pi^{+} \gamma \gamma$ branching ratio is a sensitive test of the $O\left(p^{6}\right)$ in chiral expansion. The preliminary model dependent analysis quotes:

$$
B R\left(K^{+} \rightarrow \pi^{+} \gamma \gamma\right)=\left(1.07 \pm 0.04_{\text {stat }} \pm 0.08_{\text {syst }}\right) \cdot 10^{-6}
$$

with 1164 events found on $40 \%$ of the whole data set. The shape of the $m_{\gamma \gamma}$ distribution is well described by ChPT for certain parameters values (fig.5). The process $K^{+} \rightarrow \pi^{+} e^{+} e^{-} \gamma$, never observed before, is measured in a model independent way, using 120 candidates (fig.6), with a BR of:

$$
B R\left(K^{+} \rightarrow \pi^{+} e^{+} e^{-} \gamma\right)=\left(1.19 \pm 0.12_{\text {stat }} \pm 0.04_{\text {syst }}\right) \cdot 10^{-8}
$$

This result is in perfect agreement with the $O\left(p^{6}\right)$ ChPT predictions [21]. 


\section{References}

[1] J. R. Batley et al. [NA48/2 Collaboration], Eur. Phys. J. C 52, 875 (2007) [arXiv:0707.0697 [hep-ex]].

[2] V. Fanti et al. [NA48 Collaboration], Nucl. Instrum. Meth. A 574 (2007) 433.

[3] N. Cabibbo and A. Maksymowicz, Phys. Rev. 137 (1965) B438.

[4] G. Amoros, J. Bijnens and P. Talavera, Nucl. Phys. B 585 (2000) 293 [Erratum-ibid. B 598 (2001) 665] [arXiv:hep-ph/0003258].

[5] R. Brun, R. Hagelberg, M. Hansroul and J. C. Lassalle, CERN-DD-78-2-REV (http://wwwasd.web.cern.ch/wwwasd/geant/)

[6] J. R. Batley et al. [NA48/2 Collaboration], Eur. Phys. J. C 54 (2008) 411.

[7] S. M. Roy, Phys. Lett. B 36 (1971) 353.

[8] B. Ananthanarayan, G. Colangelo, J. Gasser and H. Leutwyler, Phys. Rept. 353, 207 (2001) [arXiv:hep-ph/0005297].

[9] S. Descotes-Genon, N. H. Fuchs, L. Girlanda and J. Stern, Eur. Phys. J. C 24 (2002) 469 [arXiv:hep-ph/0112088].

[10] M. Knecht and R. Urech, Nucl. Phys. B 519 (1998) 329 [arXiv:hep-ph/9709348].

[11] B. Adeva et al. [DIRAC Collaboration], Phys. Lett. B 619 (2005) 50 [arXiv:hep-ex/0504044].

[12] G. Colangelo, J. Gasser and H. Leutwyler, Nucl. Phys. B 603 (2001) 125 [arXiv:hep-ph/0103088].

[13] C. Amsler et al. [Particle Data Group], Phys. Lett. B 667 (2008) 1.

[14] N. Cabibbo, Phys. Rev. Lett. 93 (2004) 121801 [arXiv:hep-ph/0405001].

[15] M. Zdrahal, K. Kampf, M. Knecht and J. Novotny, arXiv:0905.4868 [hep-ph].

[16] E. Gamiz, J. Prades and I. Scimemi, Eur. Phys. J. C 50 (2007) 405 [arXiv:hep-ph/0602023].

[17] N. Cabibbo and G. Isidori, JHEP 0503 (2005) 021 [arXiv:hep-ph/0502130]. Paper 1 to 1 of 1

[18] G. Colangelo, J. Gasser, B. Kubis and A. Rusetsky, Phys. Lett. B 638 (2006) 187 [arXiv:hep-ph/0604084].

[19] M. Bissegger, A. Fuhrer, J. Gasser, B. Kubis and A. Rusetsky, Nucl. Phys. B 806 (2009) 178 [arXiv:0807.0515 [hep-ph]].

[20] J. R. Batley et al. [NA48/2 Collaboration], Phys. Lett. B 633 (2006) 173 [arXiv:hep-ex/0511056].

[21] J. R. Batley et al. [NA48/2 Collaboration], Phys. Lett. B 659 (2008) 493 [arXiv:0711.4313 [hep-ex]]. 